\title{
Mitteilungen des Berufsverbands der Pneumologen in Baden- Württemberg
}

\section{Geschäftsstelle}

c/o med info $\mathrm{GmbH}$

Hainenbachstraße 25,

89522 Heidenheim

Tel 07321 9469182, Fax 073219469140

info@pneumologenverband.de,ww.pneumo-bw.de
Dr. Frank J. Heimann

(1. Vorsitzender, V.i.S. d.P.)

Dr. Michael Barczok (2. Vorsitzender)

Dr. Konrad Pumpe (Schatzmeister)

Dr. Stefan Veitshans (Schriftführer)
Dr. Erhard Bode (1. Beisitzer)

Dr. Andreas Hupert (2. Beisitzer)

Dr. Thushira Weerawarna

(Fortbildungsbeauftragter)

\section{Mitgliederversammlung digital}

Aufgrund der Corona-Pandemie fand die diesjährige Mitgliederversammlung des Landesverbandes Baden-Württemberg des Berufsverbandes der Pneumologen am 29. November virtuell im Internet statt. Mit 32 Teilnehmern nahmen in diesem Jahr mehr Pneumologinnen und Pneumologen teil als im vergangenen Jahr bei der Versammlung in der Ärztekammer in Stuttgart.

Zunächst wurden die Besonderheiten der virtuellen Versammlung sowie die Handhabung der benutzten Software dargelegt und erklärt. Insbesondere waren die anwesenden Mitglieder mit dem zur Verfügung stehenden Abstimmungsmodus einverstanden, da eine Abstimmung in geheimer Wahl nicht möglich gewesen wäre.

Inhaltlich stand die Mitgliederversammlung ganz im Zeichen des mit der AOK BadenWürttemberg sowie mit der Bosch BKK verhandelten Selektivvertrages, welcher voraussichtlich am 01.04.2021 starten wird.

Der Vorsitzende, Herr Dr. Heimann berichtete über den Verlauf der Verhandlungen mit den Krankenkassen, während Herr Dr. Barczok die Grundzüge des Vertrages bestehend aus Grundpauschalen, Einzelleistungen, Beratungsgesprächen und Qualitätszuschlägen vorstellte und auch die zu erwartende Vergütung an einzelnen Beispielen darstellte.
Herr Dr. Alexander Rupp, Beauftragter für Tabakentwöhnung im Berufsverband in BadenWürttemberg, berichtete über den erfreulich guten Stellenwert der Tabakentwöhnung im Selektivvertrag.

Als Beauftragter für digitale Pneumonie in Baden-Württemberg und im Bundesverband stellte Herr Holger Wöhrle im weiteren Verlauf der Versammlung interessante Neuentwicklungen im Bereich der Digitalisierung vor, wie die jetzt auf Rezept zu verordnenden digitalen Gesundheitsanwendungen (DiGA bzw. „App auf Rezept“). Er appellierte in diesem Zusammenhang nochmals an die Mitglieder des Landesverbandes, den Prozess der Digitalisierung aktiv mit zu gestalten, da ansonsten die Gesundheitspolitik die Digitalisierung an den Ärztinnen und Ärzten vorbei vorantreiben würde. Schon heute besteht die Möglichkeit einen substanziellen Teil der Patientenberatung/-betreuung über Videosprechstunden anbieten zu können.

In diesem Zusammenhang kritisiert der BdP Baden-Württemberg die potenzielle Möglichkeit für Krankenkassen, ihren Versicherten Apps ohne ärztliche Verordnung zur Verfügung zu stellen. Des Weiteren liegen die Kosten z.B. der vor kurzem eingeführten App Somnio zur Insomnie-Behandlung beim Vielfachen eines Regelleistungs- volumens für die ärztliche $\mathrm{Be}$ handlung/Beratung. Hier sollten zumindest ähnliche Bedingungen wie für die „analoge Versorgung" geschaffen werden. $\mathrm{Zu}$ dem fordert der BdP BaWue die im Gesetz verpflichtend vorgesehene Schaffung einer EBM Ziffer für die Beratung und Verordnung sowie Überwachung der Therapie mit einer DiGA.

Zum Schluss des Digitalisierungsteils wurde auf den am 15./16. Januar 2021 stattfindenden „BdP Digitalkonkress Pneumologie - Innovation trifft Praxis" aufmerksam gemacht und dafür eingeladen (nähere Informationen unter www.pneumologenverband.de).

Neben den inhaltlichen Diskussionen stand auch der obligatorische Rechenschaftsbericht des Schatzmeisters Herrn Dr. Pumpe sowie der Bericht der Kassenprüfer Herr Dr. Schulz und Herr Dr. Klotz auf der Tagesordnung, darüber hinaus die Entlastung des Schatzmeisters und des Vorstandes. Sowohl Herr Dr. Pumpe als Schatzmeister als auch der gesamte Vorstand wurden ohne Gegenstimmen für das vorausgehende Geschäftsjahr von den anwesenden Mitgliedern entlastet.

Auch wenn es sicherlich allen Teilnehmerinnen und Teilnehmer lieber gewesen wäre, wenn die Mitgliederversammlung als Präsenzveranstaltung hätte stattfinden können, hat die virtuelle Mitgliederversammlung sehr gut funktioniert und eine rege Diskussion und einen regen Austausch ermöglicht.

Nichtsdestotrotz hoffen wir alle, dass im kommenden Jahr die Mitgliederversammlung wieder ohne infektiologische Risiken als Präsenzveranstaltung stattfinden kann, denn neben der Veranstaltung hat so ein Treffen ja eine soziale Komponente. Pausengespräche, Kennenlernen und Kontaktpflege kommen bei einer virtuellen Veranstaltung verständlicherweise zu kurz. 